\title{
Modified lambert beer for bilirubin concentration and blood oxygen saturation prediction
}

\author{
Pek Ek Ong a,1, Audrey Kah Ching Huong a,2,*, Xavier Toh Ik Ngu ${ }^{a, 3}$, Farhanahani Mahmud a,4, \\ Sheena Punai Philimon a,5 \\ a Faculty of Electrical and Electronic Engineering, Universiti Tun Hussein Onn Malaysia, Batu Pahat, Malaysia \\ ${ }^{1}$ ong.1989@hotmail.com; ${ }^{2}$ audrey@uthm.edu.my; ${ }^{3}$ xavier@uthm.edu.my; ${ }^{4}$ farhanah@uthm.edu.my; ${ }^{5}$ ge160115@siswa.uthm.edu.my \\ * corresponding author
}

\section{ARTICLE INFO}

\section{Article history}

Received May 24, 2019

Revised June 14, 2019

Accepted June 21, 2019

Available online July 27,2019

Keywords

Bilirubin

Blood oxygen saturation

Modified lambert beer law

Raytracing

\section{ABSTRACT}

Noninvasive measurement of health parameters such as blood oxygen saturation and bilirubin concentration predicted via an appropriate light reflectance model based on the measured optical signals is of eminent interest in biomedical research. This is to replace the use of conventional invasive blood sampling approach. This study aims to investigate the feasibility of using Modified Lambert Beer model (MLB) in the prediction of one's bilirubin concentration and blood oxygen saturation value, $\mathrm{SO} 2$. This quantification technique is based on a priori knowledge of extinction coefficients of bilirubin and hemoglobin derivatives in the wavelength range of $440-500 \mathrm{~nm}$. The validity of the prediction was evaluated using light reflectance data from TracePro raytracing software for a single-layered skin model with varying bilirubin concentration. The results revealed some promising trends in the estimated bilirubin concentration with mean \pm standard deviation (SD) error of $0.255 \pm 0.025 \mathrm{~g} / \mathrm{l}$. Meanwhile, a remarkable low mean \pm SD error of $9.11 \pm 2.48 \%$ was found for the predicted SO2 value. It was concluded that these errors are likely due to the insufficiency of the MLB at describing changes in the light attenuation with the underlying light absorption processes. In addition, this study also suggested the use of a linear regression model deduced from this work for an improved prediction of the required health parameter values.

This is an open access article under the CC-BY-SA license.

\section{Introduction}

For years, the study of the use of a reflectance optical system for noninvasive monitoring of blood oxygen saturation $\left(\mathrm{SO}_{2}\right)$ and bilirubin concentration $\left(\mathrm{Cbil}_{i}\right)$ has been focused on many clinical investigations. This is mostly due to the simplicity of the system and its noninvasive attributes. Clinical monitoring of a person's $\mathrm{SO}_{2}$ is a crucial process to ensure sufficient oxygen supply to tissues throughout the body. Insufficient supply of oxygen to tissue may lead to slow wound healing process [1], headache, chest pain, rapid heartbeat and shortness of breath; in severe cases, it might also result in fatal.

Meanwhile, an elevation of bilirubin concentration in blood is a sign of jaundice or hyperbilirubinemia [2], [3]. The increase in blood bilirubin level is mostly due to poor human body excretion function to remove old red blood cells, or the breakdown product of red blood cells, from the system [4]. In a healthy circulatory system, old and damaged red blood cells would be filtered by the spleen and transported by albumin to the liver, which purpose is to eliminate these unwanted red blood cells from a human body, while new blood cells are continuously being produced in the bone marrow. 
Therefore in the cases of blockage of the bile ducts, liver disease (hepatitis) or renal failure, a high mainstream of bilirubin in blood would be ensued [5].

Bilirubin can be divided into two types, namely conjugated and unconjugated bilirubin. The unconjugated bilirubin is bounded with albumin, which would be transported to the liver. During the transferring process, the unconjugated bilirubin would be converted into conjugated bilirubin, which is water-soluble, before it was excreted from the human body through sweating and defecation system [6]. The conventional method of determining the elevation of the bilirubin level is via the observation of the yellowish of white eyes and skin. However, this method is unreliable largely due to the variation in skin color of different population. Therefore, drawing of a blood sample, or commonly known as a heel stick, is performed before the blood sample was clinically processed using blood gas analyzer. This method is tedious and time-consuming. Besides, it also increases the risk of infection at the sampling site and unnecessary blood loss.

Even though market available instrumentations such as BiliCheck (Respironics) and Minolta (part no. JM-103 by Konica Minolta/Air-shields) can reveal transcutaneous bilirubin concentration using an optical technique, the accuracy of these techniques is often subjected to differences in skin pigmentation [7], [8]. Previous studies [9]-[11] showed the viability of using diffuse reflectance spectroscopy or its hybrid imaging technologies [11] to reveal skin absorbing and scattering properties. While some of these works required the use of a biomarker in its imaging [10], others are bulky hence limiting their application to laboratory use. To this end, the use of a point reflectance system was previously proposed by Ong et al. [12] to overcome the shortcomings of the existing techniques. The common working wavelength range for the estimation of chromophores' concentration is in between $400-1000 \mathrm{~nm}$, which is known as the optical diagnostic window, due to the dominant light absorptivity of hemoglobin components and nonheme-chromophores such as bilirubin in the corresponding region [13], [14]. The prediction of the required chromophores' concentration using spectroscopy technique, however, required the use of a suitable optical model.

For this purpose, several optical models have been developed and presented in the past in the prediction of different chromophores' concentration based on light reflectance measurements [15]. The first forward model used to approximate the relationship between measurable optical signal (i.e. light absorbance) and absorptivity of the medium's absorber was Lambert Beer Model. An improvement on this model was developed by Pittman and Duling [16], who introduced a constant offset to the Lambert Beer model, to account for the effects of light scattering on the total light attenuation. The proposed model, termed as Modified Lambert Beer model (MLB), was used in the prediction of the $\mathrm{SO}_{2}$ in their in-vitro investigations. This model was later being expanded into Extended Modified Lambert Beer Law by Huong and $\mathrm{Ngu}$ [15] to allow a more accurate prediction of the $\mathrm{SO}_{2}$ and percent carbon monoxide (CO) in the blood system. Other analytical models include the cumulant based attenuation model [17], Kubelka Munk theory [18], Kramer's rule [19], Monte Carlo model [20] and cubic model [21]. Even though most of the aforementioned models were adopted to predict blood $\mathrm{SO}_{2}$, Kramer's rule [16] was designed to predict $C_{b i l}$ using a lookup table. Unlike the MLB, these previously proposed models and algorithms are computationally complex [22], involve tedious computations and are labor-intensive [23], [24]. Among the most recent advancements in the field is the use of machine learning [25], [26] in health monitoring using both supervised and unsupervised learning approaches. Such an approach, however, required a large number of datasets for data mining to improve the accuracy of its predictions.

In addition, previous works by Ong and Huong [27], Grohmann et al. [28], Danaei et al. [29] and Slusher et al. [30] showed variation in the predicted bilirubin value with differences in the selected skin site; these differences were attributed to the insufficiency of the employed optical model to describe the variation in individuals' skin pigmentation and thickness [27]. To date, to the best of the authors' knowledge, no simulation work has been done in the investigation of a suitable quantification approach in the prediction of bilirubin concentration. It is, therefore, the aim of this paper to investigate the adequacy of using MLB for this purpose. The performance of the MLB model in the prediction of health parameters namely oxygen saturation and bilirubin concentration would be evaluated based on 
the prediction error obtained from the processing of simulations from optical raytracing method (TracePro, Lambda Research Corp).

\section{Method}

\subsection{Human Skin Model}

Human skin is multilayer biological tissue having a heterogeneous distribution of particles of different size, shape, and density, both in different layers of skin and in the direction parallel to these layers, which significantly modifies light transport (via light scattering processes) in each layer in a different manner [32], [33]. This variation is further compounded with the variability in wavelength-dependent light absorption of different layers (e.g., melanin and hemoglobins in epidermal and dermal layers, respectively). The unknown specificity of skin structure, however, remains as the problems of optics of biological tissues as the former is dependent on factors such as demographic profile, skin type, body site of individuals and their gender [32], [34]. This is evident in [32], where the authors reported a good agreement in their simulation results of four-layer skin model with the works by Tuchin [35] but their results were inconsistent with previous research findings [36], [37], whose focus were on multilayer tissue of other body regions.

To this end, a significant amount of works have been published [38], [39] using monolayer skin model having homogenous optical properties to predict the basic biophysics and behavior of light-tissue interactions for application in clinical analysis and diagnosis. Not only does this simpler structure have greatly reduced cumbersome steps in numerical and analytical works [40], it helps to speed up programming time. For these reasons, this work modeled skin as a single layer medium with homogeneously distributed scattering and absorbing centers comprising of melanin, hemoglobin (oxyhemoglobin and deoxyhemoglobin) and bilirubin.

This single-layered medium of thickness $100 \mu \mathrm{m}$ and with a dimension of $1 \mathrm{~mm} \times 1 \mathrm{~mm} \times 0.1 \mathrm{~mm}$ measured from the center of the axis was modeled using TracePro (Trial version 7.7.2). The illuminating source was a fan beam with wavelengths ranging from 440 to $500 \mathrm{~nm}$ at a step size of $2 \mathrm{~nm}$. The light source was modeled to focus at a distance of $1 \mathrm{~mm}$ from coordinate $z=0$ behind the medium, as shown in Fig. 1. Meanwhile, a detector with a dimension of $0.2 \mathrm{~mm} \times 0.2 \mathrm{~mm} \times 0.1 \mathrm{~mm}$ was placed in contact with the modeled skin layer at a distance of $0.05 \mathrm{~mm}$ along the $y$-axis shown in Fig. 1 . This detector was designed as a perfect absorber to measure a number of photons backscattered from the medium.

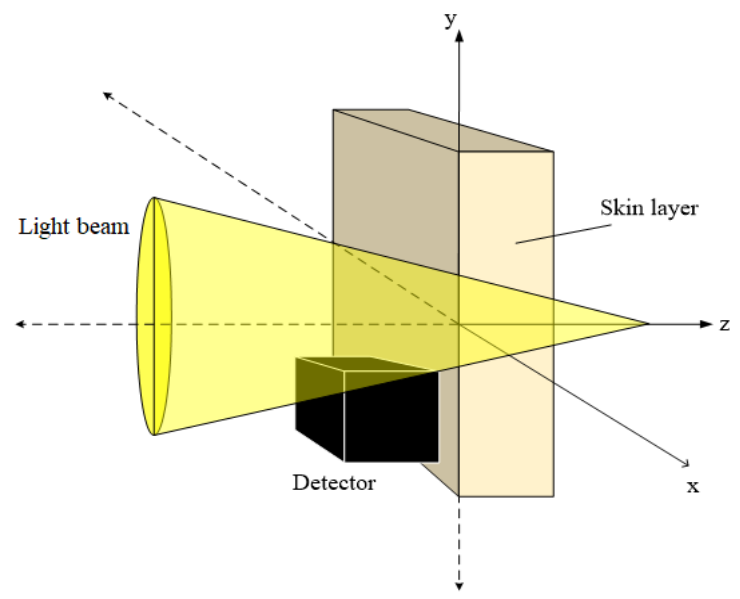

Fig. 1. The optical system designed in this work.

\subsection{Light Propagation Simulations}

In this study, the Mie scattering calculator developed by Prahl [41] was used to determine the medium's scattering coefficient based on Mie scattering model. While the sphere diameter was chosen as $0.8 \mu \mathrm{m}$, the reflective index of the sphere and the index mismatch between spheres and surrounding was chosen as 1.59 and 1.2 , respectively, to give the wavelength-dependent scattering coefficient, $\mu_{s}$, 
shown in Fig. 2. The bulk scatters properties of the medium in Fig. 1 was characterized by HenyeyGreenstein phase function [42] (a build-in tool in TracePro) based on these scattering coefficients and anisotropy factor, $g$, of 0.85 . The latter value was chosen in accordance with Anderson and Ross [43], who suggested the value should be within $0.8-1.0$, for studies of light propagation in biological tissues.

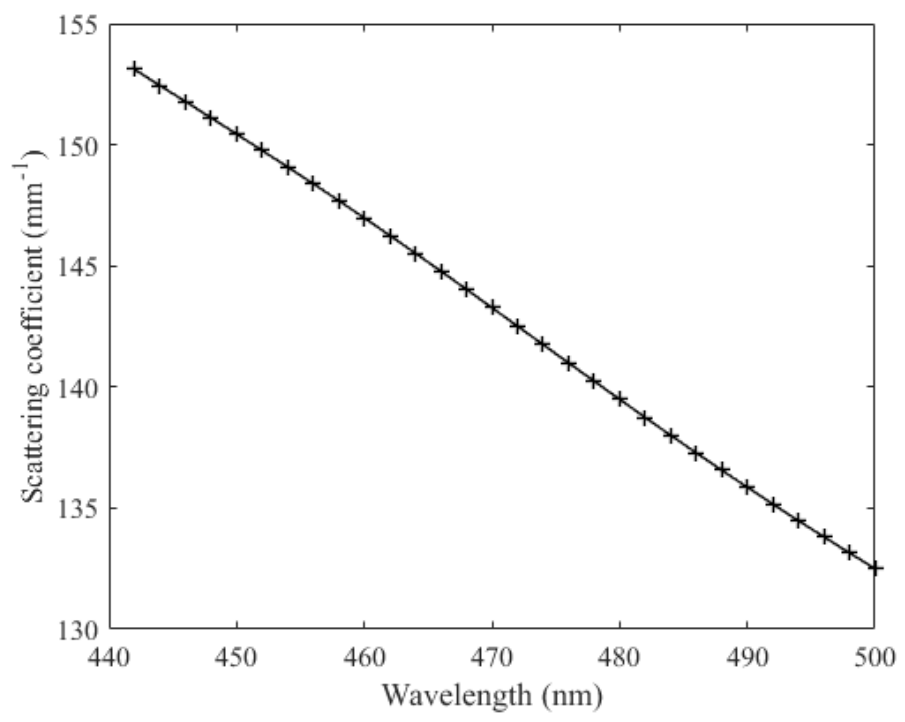

Fig. 2. The scattering coefficient, $\mu_{s}\left(\right.$ in $\mathrm{mm}^{-1}$ ), of the modeled medium.

This work assumed melanin (mel), bilirubin (bil) and hemoglobin ( $\mathrm{Hb})$, which comprised of oxyhemoglobin and deoxyhemoglobin, are the only absorbers present in the skin medium. The total absorption coefficient of the medium, $\mu$, is given by:

$$
\mu_{T}=\mu_{b i l}+\mu_{H b}+\mu_{m}
$$

where $\mu_{b i l}, \mu_{H b}$ and $\mu_{m}$ represent absorption coefficient of bil, $\mathrm{Hb}$ and mel, respectively. The absorption coefficient is given by the product of the extinction coefficient $(\varepsilon)$ and concentration $(C)$ of the absorbers present, so (1) can also be written as:

$$
\mu_{T}=\varepsilon_{b i l} C_{b i l}+\varepsilon_{o x y H b} C_{o x y H b}+\varepsilon_{d H b} C_{d H b}+\varepsilon_{m e l} C_{m e l}
$$

where the subscript oxyHb and $\mathrm{dHb}$ denote that of oxyhemoglobin and deoxyhemoglobin, respectively. The $\mu_{H b}$ in (1) can also be expressed as [1]:

$$
\mu_{H b}=\left(\left[\varepsilon_{o x y H b}-\varepsilon_{d H b}\right] S O_{2}+\varepsilon_{d H b}\right) T .
$$

In this work, $\mathrm{SO}_{2}$ was assumed as a constant with a percent value of $98 \%$ while total blood concentration, $T$, is taken here as $150 \mathrm{~g} / \mathrm{l}$. It was reported in Ong and Huong [27] that the wavelengthdependent absorption of melanin, $\mu_{m}$, is given by (4). The wavelength-dependent extinction coefficient of other considered absorbers are taken from the Oregon Medical Laser Center website [41] shown in Fig. 3.

$$
\mu_{m}=5 \times 10^{9} \lambda^{-3.33} .
$$

This study considered only signals in the wavelength range of $440-500 \mathrm{~nm}$ owing to the peak bilirubin absorptivity at this range. The simulation works were carried out for medium with five arbitrarily chosen bilirubin concentration values, $C_{b i l}$, namely $0.32 \mathrm{~g} / 1,0.38 \mathrm{~g} / 1,0.45 \mathrm{~g} / 1,0.51 \mathrm{~g} / \mathrm{l}$, and $0.64 \mathrm{~g} / \mathrm{l}$. Substituting $C_{b i l}$ into (2) to give the five different ranges of wavelength varying light absorptivity. Shown in Fig. 4 are the simulation results for the scattering-absorbing medium of the considered varying $C_{b i l}$. 


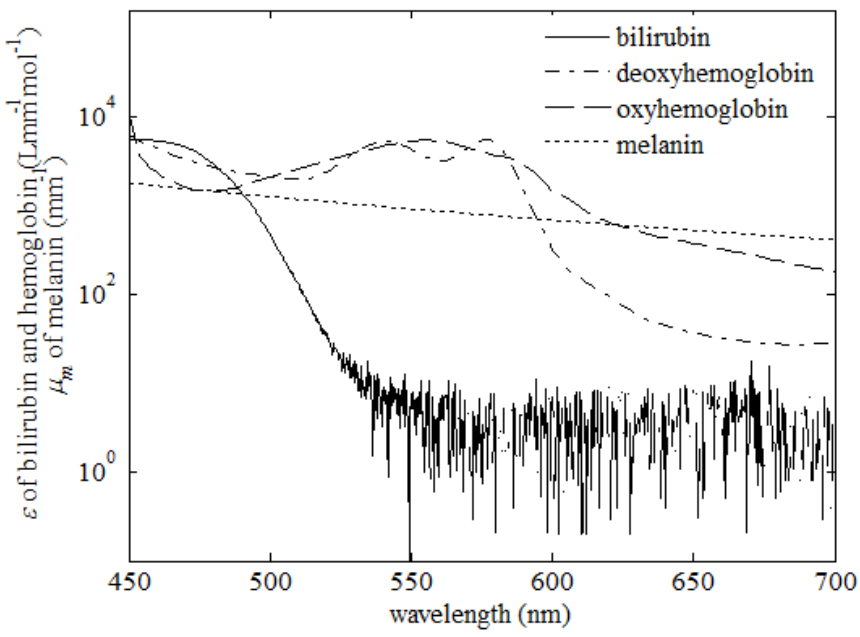

Fig. 3. Wavelength dependent extinction coefficient of the absorbers considered in this study. Data were taken from [41], [44], and [45].

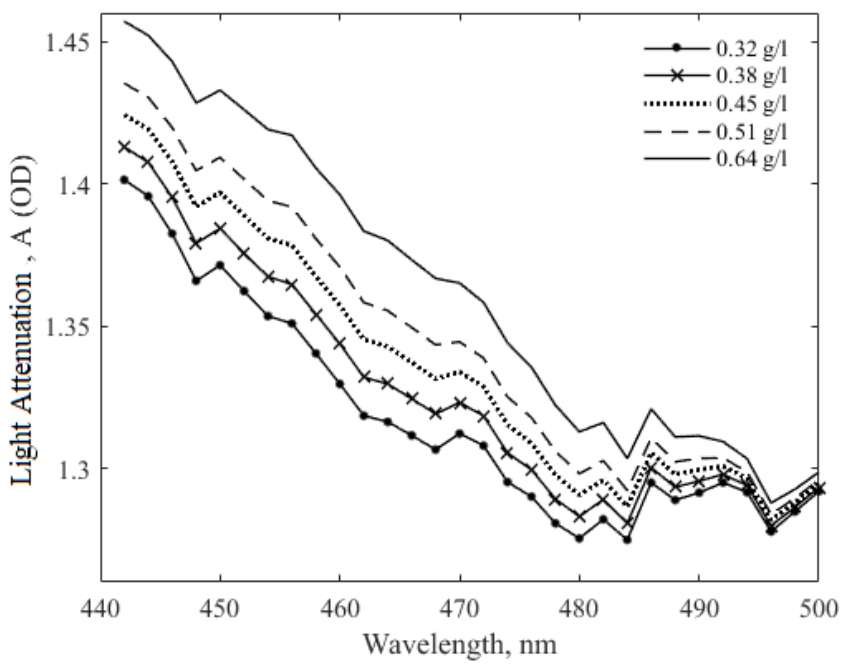

Fig. 4. The wavelength-dependent light attenuation simulated using TracePro for the medium of different bilirubin concentration value, $C_{b i l}$.

\subsection{Modified Lambert Beer Model and Fitting Procedure}

In this work, the MLB model proposed by Pittman and Duling [16] was employed to give the best guess of the medium's $\mathrm{C}_{b i}$ and $\mathrm{SO}_{2}$. This model is comparatively simple, easy to understand, and required less computational power as compared to other models described in section 1. This model assumed a linear relationship between the measured light attenuation and the medium's absorption as followed:

$$
A(\lambda)=G+\mu_{a} d
$$

where $G$ is a constant value representing scattering dependent light attenuation offset while $d$ is normally taken as the mean light pathlength. Since changes in melanin absorptivity across the considered wavelengths are relatively small as shown in Fig. 3, it was assumed that its effects on the total light attenuation is taken care of by parameter $G$. This reduces the $\mu_{a}$ in (5) to that given by bilirubin and hemoglobin components in the medium. Thus, substituting (2) into (5) gives:

$$
A(\lambda)=G+\varepsilon_{b i l} C_{b i l} d+\left(\left[\varepsilon_{o x y H b}-\varepsilon_{d H b}\right] S O_{2}+\varepsilon_{d H b}\right) d T .
$$

The prediction of the unknowns in (6) (i.e. $G, \mathrm{Cb}_{b i l}, \mathrm{SO}_{2}$ and $d$ ) was via an iterative fitting routine using fminsearch function available in MATLAB (version 2016a). This was accomplished by fitting the 
simulations (of different $C_{b i l}$ range of $0.32-0.64 \mathrm{~g} / 1$ shown in Fig. 4) using the MLB in (6). This process required the extinction coefficients of bilirubin and hemoglobin components in the wavelength range of $440-500 \mathrm{~nm}$ as its priori knowledge following the equation in (6). The fitting process began by initializing all the unknowns with value ' 1 ', the optimal value was iteratively sought based on the absolute difference between the value given by the simulated attenuation and the values given by MLB, $\triangle E$. This fitting procedure ended when either the number of iteration has reached 500 or $\Delta E$ is less than $10^{-20}$.

\section{Results and Discussion}

Based on the steps discussed in section 2.3, results from TracePro simulations for single-layered scattering-absorbing medium were used to investigate the feasibility of using the MLB model in the estimation of the medium's $\mathrm{C}_{b i l}$ and $\mathrm{SO}_{2}$.

The $\mathrm{SO}_{2}$ and $C_{b i l}$ value predicted using MLB for different $C_{b i l}$ condition is shown in Table 1, they are represented in the following by the symbol $S_{2}(p)$ and $C_{b i l}(p)$, respectively. Fig. 5 shows a plot comparing the ground truth with the predicted bilirubin concentration value for a different set of simulation data, while Fig. 6 shows the relationship between the results in Fig. 5. Also shown in this diagram is the best fitted linear regression equation for the relationship.

Table 1. The predicted values, $C_{b i l}(p)$ and $S_{2(p)}$, for different $C_{b i l}$ used in the simulation. The ground truth of $\mathrm{SO}_{2}$ value is given by $98 \%$.

\begin{tabular}{ccc}
\hline Bilirubin & \multicolumn{2}{c}{ Predicted values } \\
\cline { 2 - 3 } concentration, $_{\boldsymbol{C}_{\boldsymbol{b i l}}(\mathrm{g} / \mathbf{l})}$ & $\begin{array}{c}\text { Bilirubin concentration, } \\
\boldsymbol{C}_{\boldsymbol{b i l}(\boldsymbol{p})}(\mathrm{g} / \mathbf{l})\end{array}$ & $\begin{array}{c}\text { Blood oxygen saturation, } \\
\boldsymbol{S O}_{\mathbf{2}(\boldsymbol{p})}(\%)\end{array}$ \\
\hline 0.32 & 0.0363 & 106.89 \\
0.38 & 0.1131 & 107.09 \\
0.45 & 0.1887 & 111.22 \\
0.51 & 0.2613 & 105.61 \\
0.64 & 0.4236 & 104.76 \\
\hline
\end{tabular}

Based on Table 1, the mean and standard deviation (SD) of the absolute error in the predictions of bilirubin concentration is calculated as $0.255 \pm 0.025 \mathrm{~g} / 1$ while the mean $\pm \mathrm{SD}$ of error in the predicted percent oxygen saturation is given by $9.11 \pm 2.48 \%$. Even though noticeable differences in the value of $C_{b i l}(p)$ as compared to that used in the simulations was found in Table 1, the result in Fig. 5 revealed some promising trends in the predicted values. It can be observed from Fig. 5 that the increase in $C_{b i l}(p)$ with the attenuation index is of the same rate and fashion as that of the ground truth value. This is evident with the considerably linear relationship between the actual and predicted value shown in Fig. 6. This relationship between the actual and the predicted bilirubin concentration, $C_{b i l}(p)$, listed in Table 1 was further evaluated using the correlation test in MATLAB. The calculated correlation coefficient value of 0.9997 showed a relatively strong association between the two variables. Thus, suggesting the feasibility of using a linear regression model shown in Fig. 6 to correct for the predicted value.

It must also be noted that the differences between the ground truth and the predicted bilirubin concentration values reduced steadily with the increase in light attenuation index (i.e. increase in medium's bilirubin concentration) in Table 1 . This may imply an increase in the adequacy of the employed MLB to describe changes in measurable output with variations in the medium's optical properties under high bilirubin level conditions.

There are several factors contributing to the error in the $C_{b i l}(p)$ shown in Fig. 5. Among them include the effects of wavelength varying melanin and light scattering properties shown in Fig. 2 and Fig. 3 on the measured light attenuation, which have not accounted for in the employed forward model in (6). In addition, the wavelength varying scattering coefficients would have different influence on the amount of light being backscattered onto the detector at each wavelength; therefore it is inappropriate to represent these effects with a constant parameter $G$ in (6). This, together with the wavelength-dependent 
absorptivity of the medium's absorbers, would also modify the light path-length value, $d$, in (6) at different wavelength [46], [47].

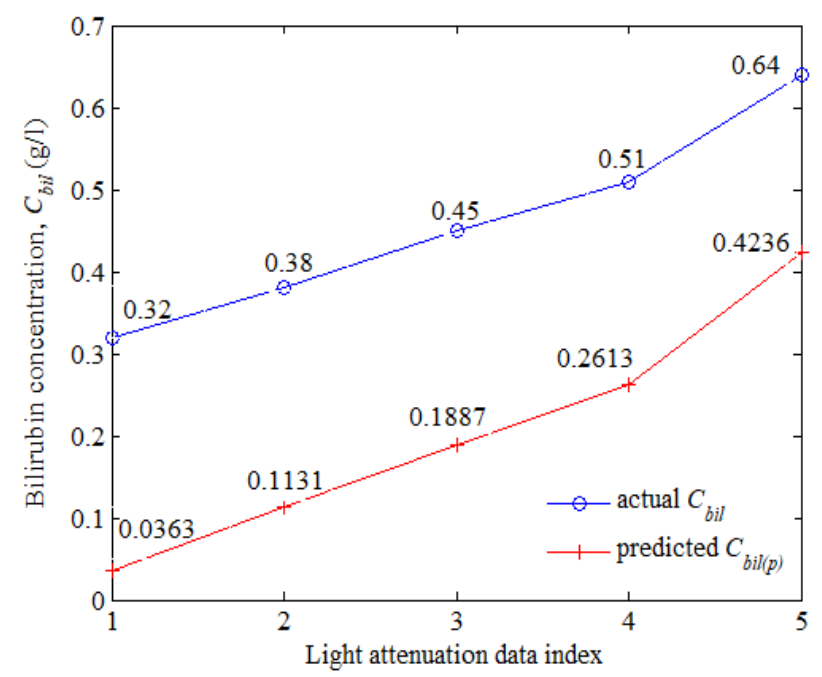

Fig. 5. A comparison between the actual and bilirubin concentration, $C_{b i l}(p)$, predicted using data from TracePro.

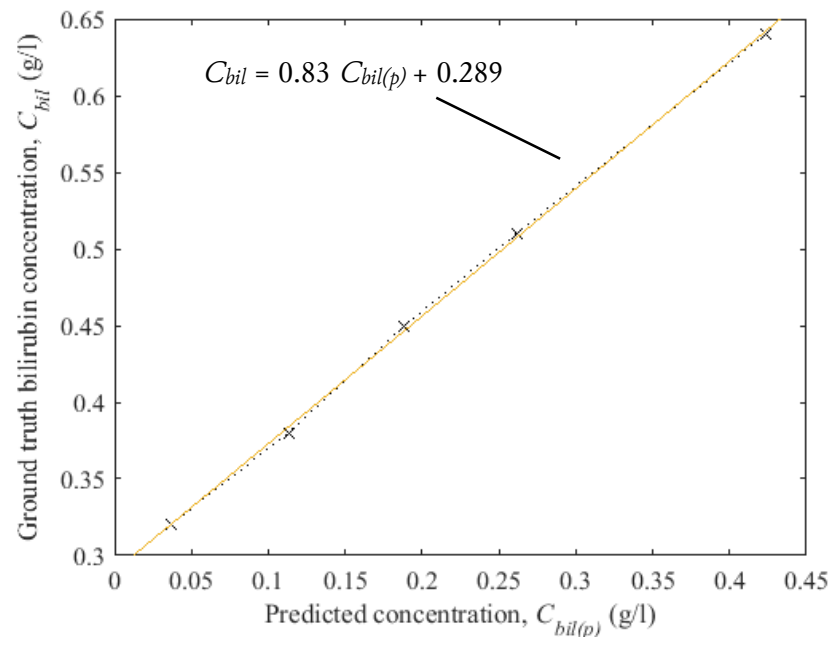

Fig. 6. The relationship between the actual and the predicted value, $C_{b i l}(p)$ (dotted line with cross symbols). Also shown here is the best fitted linear regression line.

It is worth noting that the $\mathrm{SO}_{2}$ predicted using the MLB appeared to be considerably robust towards changes in the optical properties in the medium. Even though the corresponding results are favorable, from the discussions in the earlier paragraph, this could indicate the possibility of overfitting in the recruited algorithm described in section 2.3. Since the MLB has limitation in its prediction performances, further work includes the use of neural network $(\mathrm{NN})$ approach to overcome the problem of finding a suitable optical model for quantification purposes. Nonetheless, appropriate features, extracted from a large number of datasets such as that shown in Fig. 4, would be needed in the training of the NN.

\section{Conclusion}

This work demonstrated the use of MLB model in the estimation of Cbil and SO2. This study observed noticeable discrepancies in the prediction of bilirubin concentration with error given by 0.255 $\pm 0.025 \mathrm{~g} / \mathrm{l}$. Even so, this study found some promising trends in the predicted values and a relatively strong correlation between the actual and predicted Cbil. Besides, the results revealed a high consistency 
and relatively low mean error in the predicted mean SO2 given by $9.11 \pm 2.48 \%$. This study concluded that a linear regression model determined from the ground-truth and bilirubin concentration prediction relationship may be potentially used in the future for the quantification work. In addition, the use of $\mathrm{NN}$ system may be a potential alternative in the prediction works.

\section{Acknowledgment}

The authors are thankful to Lambda Research for providing the Trial version of TracePro software. We are also grateful to Universiti Tun Hussein Onn Malaysia under GPPS grant num. U583 and U935, and Ministry of Education Malaysia (under FRGS grant num. 1581) for financially supporting this work.

\section{References}

[1] A. Huong, S. Philimon, and X. Ngu, "Multispectral imaging of acute wound tissue oxygenation," Journal of Innovative Optical Health Sciences, vol. 10, p. 1750004, 2017, doi: 10.1142/S1793545817500043.

[2] J. M. Kirk, "Neonatal jaundice: a critical review of the role and practice of bilirubin analysis," Annals of clinical biochemistry, vol. 45, pp. 452-462, 2008, doi: 10.1258/acb.2008.008076.

[3] S. K. Alla, A. Huddle, J. D. Butler, P. S. Bowman, J. F. Clark, and F. R. Beyette, "Point-of-care device for quantification of bilirubin in skin tissue," IEEE Transactions on Biomedical Engineering, vol. 58, pp. 777780, 2011, doi: 10.1109/TBME.2010.2093132.

[4] S. A. Kumar, P. R. Bhadri, F. Beyette, J. F. Clark, and W. Wurster, "Non-invasive biomedical system for the quantification of bilirubin in neonates," in 48th Midwest Symposium on Circuits and Systems, 2005., 2005, pp. 1778-1781, doi: 10.1109/MWSCAS.2005.1594466.

[5] N. Polley, S. Saha, S. Singh, A. Adhikari, S. Das, B. R. Choudhury, et al., "Development and optimization of a noncontact optical device for online monitoring of jaundice in human subjects," Journal of biomedical optics, vol. 20, p. 067001, 201, doi: 10.1117/1.JBO.20.6.067001.

[6] Z. Zulkarnay, A. Jurimah, B. Ibrahim, S. Shazwani, E. Cheng, A. Ruzairi, et al., "An overview on jaundice measurement and application in biomedical: The potential of non-invasive method," in 2015 2nd International Conference on Biomedical Engineering (ICoBE), 2015, pp. 1-6, doi: 10.1109/ICoBE.2015.7235896.

[7] P. M. Varughese and L. Krishnan, "Does color really matter? Reliability of transcutaneous bilirubinometry in different skin-colored babies," Indian Journal of Paediatric Dermatology, vol. 19, p. 315, 2018, doi: 10.4103/ijpd.IJPD_3_18.

[8] S. Samiee-Zafarghandy, J. Feberova, K. Williams, A. Yasseen, S. Perkins, and B. Lemyre, "Influence of skin colour on diagnostic accuracy of the jaundice meter JM 103 in newborns," Archives of Disease in ChildhoodFetal and Neonatal Edition, vol. 99, pp. F480-F484, 2014, doi: 10.1136/archdischild-2013-305699.

[9] N. Verdel, A. Marin, M. Milanič, and B. Majaron, "Physiological and structural characterization of human skin in vivo using combined photothermal radiometry and diffuse reflectance spectroscopy," Biomedical Optics Express, vol. 10, pp. 944-960, 2019, doi: 10.1364/BOE.10.000944.

[10] T. M. Bydlon, R. Nachabé, N. Ramanujam, H. J. Sterenborg, and B. H. Hendriks, "Chromophore based analyses of steady-state diffuse reflectance spectroscopy: current status and perspectives for clinical adoption," Journal of biophotonics, vol. 8, pp. 9-24, 2015, doi: 10.1002/jbio.201300198.

[11] C. Veenstra, W. Petersen, I. M. Vellekoop, W. Steenbergen, and N. Bosschaart, "Spatially confined quantification of bilirubin concentrations by spectroscopic visible-light optical coherence tomography," Biomedical optics express, vol. 9, pp. 3581-3589, 2018, doi: 10.1364/BOE.9.003581.

[12] P. Ong, A. K. Huong, W. Hafizah, K. Tay, and S. P. Philimon, "Reflectance spectroscopy system for noninvasive prediction of skin bilirubin concentration related parameter," in 2016 IEEE EMBS Conference on Biomedical Engineering and Sciences (IECBES), 2016, pp. 352-355, doi: 10.1109/IECBES.2016.7843472.

[13] R. B. Saager, M. L. Baldado, R. A. Rowland, K. M. Kelly, and A. J. Durkin, "Method using in vivo quantitative spectroscopy to guide design and optimization of low-cost, compact clinical imaging devices: emulation and evaluation of multispectral imaging systems," Journal of biomedical optics, vol. 23, p. 046002, 2018, doi: 10.1117/1.JBO.23.4.046002. 
[14] F. Vasefi, N. MacKinnon, R. B. Saager, A. J. Durkin, R. Chave, E. H. Lindsley, et al., "Polarization-sensitive hyperspectral imaging in vivo: a multimode dermoscope for skin analysis," Scientific reports, vol. 4, p. 4924, 2014, doi: 10.1038/srep04924.

[15] A. Huong and X. Ngu, "The application of Extended Modified Lambert Beer model for measurement of blood carboxyhemoglobin and oxyhemoglobin saturation," Journal of Innovative Optical Health Sciences, vol. 7, 2014, doi: 10.1142/S1793545814500266.

[16] R. N. Pittman and B. R. Duling, "A new method for the measurement of percent oxyhemoglobin," Journal of applied physiology, vol. 38, pp. 315-320, 1975, doi: 10.1152/jappl.1975.38.2.315.

[17] A. K. C. Huong, "Spectroscopic analysis of scattering media via different quantification techniques," Thesis, University of Nottingham, 2012, available at: Google Scholar.

[18] S. J. Madsen and B. C. Wilson, "Optical properties of brain tissue," in Optical methods and instrumentation in brain imaging and therapy, ed: Springer, 2013, pp. 1-22, doi: 10.1007/978-1-4614-4978-2_1.

[19] N. Ali and Z. Z. Abidin, "A Review of Non-Invasive Jaundice detection using Optical Technique in Neonates," in Conference in Advances In Computing, Electronics and Electrical Technology-CEET 2014, 2014, pp. I-3, available at: Google Scholar.

[20] A. Bjorgan, M. Milanic, and L. L. Randeberg, "Estimation of skin optical parameters for real-time hyperspectral imaging applications," Journal of biomedical optics, vol. 19, p. 066003, 2014, doi: 10.1117/1.JBO.19.6.066003.

[21] M. Hirose, M. Kuroshima, and N. Tsumura, "Nonlinear estimation of chromophore concentrations, shading and surface reflectance from five band images," in Color and Imaging Conference, 2015, pp. 161-166, available at: Google Scholar.

[22] G. Einstein, P. Aruna, and S. Ganesan, "Monte Carlo based model for diffuse reflectance from turbid media for the diagnosis of epithelial dysplasia," Optik, vol. 181, pp. 828-835, 2019, doi: 10.1016/j.ijleo.2018.12.158.

[23] A. Moy and J. Tunnell, "Diffuse Reflectance Spectroscopy and Imaging," in Imaging in Dermatology, ed: Elsevier, 2016, pp. 203-215, doi: 10.1016/B978-0-12-802838-4.00017-0.

[24] L. Hou, Y. Liu, L. Qian, Y. Zheng, J. Gao, W. Cao, et al., "Portable Near-Infrared Technologies and Devices for Noninvasive Assessment of Tissue Hemodynamics," Journal of Healthcare Engineering, vol. 2019, 2019, doi: 10.1155/2019/3750495.

[25] Y. Karamavuş and M. Özkan, "Newborn jaundice determination by reflectance spectroscopy using multiple polynomial regression, neural network, and support vector regression," Biomedical Signal Processing and Control, vol. 51, pp. 253-263, 2019, doi: 10.1016/j.bspc.2019.01.019.

[26] E. E. Tripoliti, T. G. Papadopoulos, G. S. Karanasiou, K. K. Naka, and D. I. Fotiadis, "Heart failure: diagnosis, severity estimation and prediction of adverse events through machine learning techniques," Computational and structural biotechnology journal, vol. 15, pp. 26-47, 2017, doi: 10.1016/j.csbj.2016.11.001.

[27] P. Ong and A. K. Huong, "Point Spectroscopy System for Noncontact and Noninvasive Prediction of Transcutaneous Bilirubin Concentration," in IOP Conference Series: Materials Science and Engineering, 2017, p. 012130, doi: 10.1088/1757-899X/226/1/012130.

[28] K. Grohmann, M. Roser, B. Rolinski, I. Kadow, C. Müller, A. Goerlach-Graw, et al., "Bilirubin measurement for neonates: comparison of 9 frequently used methods," Pediatrics, vol. 117, pp. 1174-1183, 2006, doi: 10.1542/peds.2005-0590.

[29] N. Danaei, R. Ghorbani, A. Emadi, and S. Nooripour, "Evaluating the diagnostic value of skin bilirubin in comparison with plasma bilirubin to identify hyperbilirubinemia in healthy babies," Middle East Journal of Rehabilitation and Health, vol. 3, 2016, doi: 10.17795/mejrh-33493.

[30] T. M. Slusher, I. A. Angyo, F. Bode-Thomas, F. Akor, S. D. Pam, A. A. Adetunji, et al., "Transcutaneous bilirubin measurements and serum total bilirubin levels in indigenous African infants," Pediatrics, vol. 113, pp. 1636-1641, 2004, doi: 10.1542/peds.113.6.1636.

[31] S. J. Jo and O. S. Kwon, "Structure and Function of Skin: The Application of THz Radiation in Dermatology," in Convergence of Terahertz Sciences in Biomedical Systems, ed: Springer, 2012, pp. 281-299, doi: 10.1007/978-94-007-3965-9_16. 
[32] A. Bhandari, B. Hamre, Ø. Frette, K. Stamnes, and J. Stamnes, "Modeling optical properties of human skin using Mie theory for particles with different size distributions and refractive indices," Optics express, vol. 19, pp. 14549-14567, 2011, doi: 10.1364/OE.19.014549.

[33] S. L. Jacques, "Optical assessment of tissue heterogeneity in biomaterials and implants," in Laser-Tissue Interaction XI: Photochemical, Photothermal, and Photomechanical, 2000, pp. 576-581, doi: 10.1117/12.388080.

[34] J. A. Iglesias-Guitian, C. Aliaga, A. Jarabo, and D. Gutierrez, "A biophysically-based model of the optical properties of skin aging," in Computer Graphics Forum, 2015, pp. 45-55, doi: 10.1111/cgf.12540.

[35] V. V. Tuchin, I. L. Maksimova, D. A. Zimnyakov, I. L. Kon, A. H. Mavlyutov, and A. A. Mishin, "Light propagation in tissues with controlled optical properties," Journal of biomedical optics, vol. 2, pp. 401-418, 1997, doi: 10.1117/1.429841.

[36] H. Ding, J. Q. Lu, W. A. Wooden, P. J. Kragel, and X.-H. Hu, "Refractive indices of human skin tissues at eight wavelengths and estimated dispersion relations between 300 and $1600 \mathrm{~nm}$," Physics in Medicine \& Biology, vol. 51, p. 1479, 2006, doi: 10.1088/0031-9155/51/6/008.

[37] J.-C. Lai, Y.-Y. Zhang, Z.-H. Li, H.-J. Jiang, and A.-Z. He, "Complex refractive index measurement of biological tissues by attenuated total reflection ellipsometry," Applied optics, vol. 49, pp. 3235-3238, 2010, doi: 10.1364/AO.49.003235.

[38] S. Chatterjee, J. Phillips, and P. Kyriacou, "Monte Carlo investigation of the effect of blood volume and oxygen saturation on optical path in reflectance pulse oximetry," Biomedical Physics \& Engineering Express, vol. 2, p. 065018, 2016, doi: 10.1088/2057-1976/2/6/065018.

[39] I. Krasnikov, C. Suhr, A. Seteikin, B. Roth, and M. Meinhardt-Wollweber, "Two efficient approaches for modeling of Raman scattering in homogeneous turbid media," JOSA A, vol. 33, pp. 426-433, 2016, doi: 10.1364/JOSAA.33.000426.

[40] V. Barun and A. Ivanov, "Role of epidermis in the optics and thermal physics of human skin," Optics and Spectroscopy, vol. 107, p. 909, 2009, doi: 10.1134/S0030400X09120121.

[41] S. Prahl, "Optical absorption of hemoglobin," 1999, available at: https://omlc.org/spectra/hemoglobin/.

[42] L. G. Henyey and J. L. Greenstein, "Diffuse radiation in the galaxy," The Astrophysical Journal, vol. 93, pp. 70-83, 1941, doi: 10.1086/144246.

[43] R. Anderson and E. Ross, "Laser-tissue interactions," Cutaneous Laser Surgery: The Art and Science of Selective Photo-thermolysis. Mosby-Year Book: St Louis, pp. 1-18, 1994, available at: Google Scholar.

[44] G. Agati and F. Fusi, "New trends in photobiology recent advances in bilirubin photophysics," Journal of Photochemistry and Photobiology B: Biology, vol. 7, pp. 1-14, 1990, doi: 10.1016/1011-1344(90)85138-M.

[45] I. V. Meglinski and S. J. Matcher, "Quantitative assessment of skin layers absorption and skin reflectance spectra simulation in the visible and near-infrared spectral regions," Physiological Measurement, vol. 23, p. 741, 2002, doi: 10.1088/0967-3334/23/4/312.

[46] T. Kitai, A. Tanaka, A. Tokuka, K. Tanaka, Y. Yamaoka, K. Ozawa, et al., "Quantitative detection of hemoglobin saturation in the liver with near-infrared spectroscopy," Hepatology, vol. 18, pp. 926-936, 1993, doi: 10.1002/hep.1840180426.

[47] D. Rimini, F. Molinari, W. Liboni, M. Balbo, R. Darò, E. Viotti, et al., "Effect of ocular movements during eye movement desensitization and reprocessing (EMDR) therapy: a near-infrared spectroscopy study," PloS one, vol. 11, p. e0164379, 2016, doi: 10.1371/journal.pone.0164379. 